\title{
Avaliação epidemiológica dos pacientes vítimas de queimadura ocular pelo agente químico cal no Serviço de Oftalmologia do Hospital Universitário Evangélico de Curitiba
}

\author{
Epidemiological assessment at the Ophthalmology Departamentof the Evangelic \\ University Hospital of Curitiba of patients victims of ocularlime burns
}

\author{
Alessandra Guerra Daros Castellano ${ }^{1}$ \\ Hamilton Moreira ${ }^{2}$ \\ Rommel Josué Zagó \\ Faye Salvatierra Milicovsky ${ }^{4}$
}

${ }^{1}$ Médica Oftalmologista do Hospital Universitário Evangélico de Curitiba.

${ }^{2}$ Coordenador da Residência de Oftalmologia do Hospital Universitário Evangélico de Curitiba.

${ }^{3}$ Residente de Oftalmologia do Hospital Universitário Evangélico de Curitiba.

${ }^{4}$ Médica estagiária do Departamento de Oftalmologia do Hospital Universitário Evangélico de Curitiba.

Endereço para correspondência: R. Capitão Souza Franco, 833, apto. 41 - Curitiba (PR) CEP 80730-420. E-mail: maurocastellano@uol.com.br

Recebido para publicação em 28.05.2001 Aceito para publicação em 16.01.2002

Nota Editorial: Pela análise deste trabalho e por sua anuência sobre a divulgação desta nota, agradecemos ao Dr. Luiz Antonio Vieira.

\section{RESUMO}

Objetivo: Avaliar o perfil epidemiológico dos pacientes vítimas de queimadura ocular pelo agente químico cal, no Serviço de Oftalmologia do Hospital Universitário Evangélico de Curitiba, enfatizando-se os casos de queimaduras oculares pela "Bomba de Cal". Métodos: Estudo prospectivo de 88 pacientes com queimadura ocular pela cal (cal sem explosão ou bomba de cal), que procuraram o serviço de Pronto Atendimento do referido hospital no período de setembro de 1999 a setembro de 2000. Resultados: Dos 88 pacientes avaliados $73(82,95 \%)$ foram vítimas de queimadura ocular pela cal no ambiente de trabalho e 15 (17,24\%) foram vítimas da "Bomba de Cal" (brincadeira regional). A maioria dos pacientes examinados foi do sexo masculino, com faixa etária entre 20 e 40 anos, com predomínio de queimadura ocular unilateral e grau I quando vítimas de acidente de trabalho e bilateral e grau IV quando a queimadura foi resultante da "Bomba deCal". Conclusão: Comparando-se os grupos estudados observou-se que o grupo vítima de queimadura ocular pela "Bomba de Cal" apresentou maior agravo à saúde ocular demonstrado, neste estudo, pela bilateralidade, gravidade das lesões e idade mais precoce de acometimento dos pacientes.

Descritores: Queimaduras oculares; Olho/efeitos de drogas; Queimaduras químicas: Álcalis/efeitos adversos; Acidentes de trabalho/prevenção e controle; Serviço hospitalar de emergência; Hospitais universitários

\section{INTRODUÇÃO}

As queimaduras químicas oculares são causa freqüente de danos à saúde ocular e devem ser consideradas emergências oftalmológicas devido à sua alta morbidade. São freqüentemente bilaterais e potencialmente devastadoras acometendo 7 a $10 \%$ dos casos relatados de traumas oculares ${ }^{(1-4)}$.

A literatura tem demonstrado que dentre os agentes químicos causadores de queimaduras oculares, as substâncias alcalinas são as mais freqüentes e são responsáveis por graves seqüelas à saúde ocular ${ }^{(5-9)}$. A maioria das vítimas é do sexo masculino, jovem e observa-se alta incidência de acidentes de trabalho ${ }^{(1,4-11)}$.

A gravidade da lesão ocular depende da concentração do agente agressor, da duração da exposição do globo ocular ao agente, do pH da solução e da velocidade de penetração da droga $^{(3)}$. As lesões por álcali têm como agentes mais freqüentes: amônia $\left(\mathrm{NH}_{3}\right)$ soda cáustica $(\mathrm{NaOH})$, cal $(\mathrm{Ca}$ $\left.(\mathrm{OH})_{2}\right)$ e hidróxido de potássio $(\mathrm{KOH})$. Os álcalis são comumente mais 
destrutivos quando comparados aos ácidos ${ }^{(12)}$, caracterizando-se pela rapidez com que penetram no olho induzindo a alterações teciduais. $\mathrm{O}$ ânion hidróxido $\left(\mathrm{OH}^{-}\right)$saponifica os ácidos gordurosos das membranas celulares, com resultante rotura e morte celular ${ }^{(13-15)}$, e o cátion é responsável pela penetração do álcali específico ${ }^{(15)}$.

Cátions reagem com os grupos carboxílicos $\left(\mathrm{COOH}^{-}\right)$do colágeno estromal e dos glicosaminoglicans e a hidratação destes, resulta em perda de transparência do estroma corneano. A hidratação das fibrilas colágenas provoca o espessamento e encurtamento destas, o que distorce o trabeculado escleral, predispondo à liberação de prostaglandinas que acabam por aumentar a pressão intraocular imediatamente após a queimadura química ${ }^{(16)}$.

Quando há contato de compostos alcalinos em quantidade suficiente na superfície ocular, em 3 a 5 minutos encontra-se a substância alcalina no humor aquoso ${ }^{(17)}$, ocorrendo, portanto, comprometimento não somente da superfície ocular, mas também do estroma, endotélio corneano e estruturas intra-oculares como íris, cristalino, trabeculado e corpo ciliar ${ }^{(13)}$.

Os tecidos sãos, adjacentes à lesão, determinam uma inflamação reacional e uma quimiotaxia para leucócitos polimorfonucleares, causando a liberação de enzimas lisossomiais marcadas (colagenose), que tem atividade colagenolítica podendo levar a complicações corneanas graves como ulceração e perfuração corneana ${ }^{(18)}$.

As queimaduras químicas oculares, sobretudo as devido ao agente químico cal, têm sido causa freqüente de injúrias oculares no Serviço de Oftalmologia do Hospital Universitário Evangélico de Curitiba - HUEC. Agravando este quadro, aparece a "Bomba de Cal", uma brincadeira regional que é feita por crianças que inocentemente vêem a explosão como uma diversão.

A "Bomba de Cal" é confeccionada adicionando-se, água e certa quantidade de cal virgem, a uma garrafa de plástico descartável. Com o fechamento da garrafa e a interação de tais elementos, ocorre uma reação química com aumento de pressão no interior da garrafa, que culmina com a explosão da mesma, causando o contato do composto alcalino com as pessoas dentro do raio de ação da bomba.

Considerando-se o exposto acima, este estudo pretende avaliar o perfil epidemiológico dos pacientes vítimas de queimadura química ocular pelo agente cal no Serviço de Oftalmologia do HUEC, enfatizando-se os casos de queimadura ocular pela "Bomba de Cal" e alertar a comunidade científica e a sociedade sobre as conseqüências deste tipo de lesão ocular.

\section{MÉTODOS}

Foi realizado um estudo prospectivo com 88 pacientes que apresentaram queimadura química ocular pela cal, que procuraram o Pronto Atendimento Oftalmológico do Hospital Universitário Evangélico de Curitiba, no período de setembro de 1999 a setembro de 2000.

Conforme protocolo elaborado para o trabalho, os pacientes foram avaliados quanto ao sexo, faixa etária, agente causal (cal sem explosão ou "Bomba de Cal"), lateralidade do acometimento ocular e gravidade da queimadura química ocular segundo a classificação para queimadura química proposta por Ballen (1964) e Hopper-Hall (1965) ${ }^{(19)}$. (Tabela 1)

\section{RESULTADOS}

Durante o período do estudo foram atendidos 88 pacientes portadores de queimadura química ocular pelo agente cal no Pronto Atendimento de Oftalmologia do HUEC, sendo 73 $(82,95 \%)$ pacientes vítimas de queimadura ocular pela cal sem explosão e 15 (17,05\%) pacientes vítimas da "Bomba de Cal".

$\mathrm{Na}$ análise dos casos de queimadura ocular pela cal sem explosão (73-82,95\%) pôde-se evidenciar maior incidência no sexo masculino (69-94,52\%) com faixa etária predominando entre 20 e 40 anos, sendo que a maioria dos casos ocorreu no ambiente de trabalho (Figura 1). O acometimento ocular ocorreu em 87 olhos, sendo bilateral em $14(19,18 \%)$ pacientes e unilateral em $59(80,82 \%)$ pacientes.

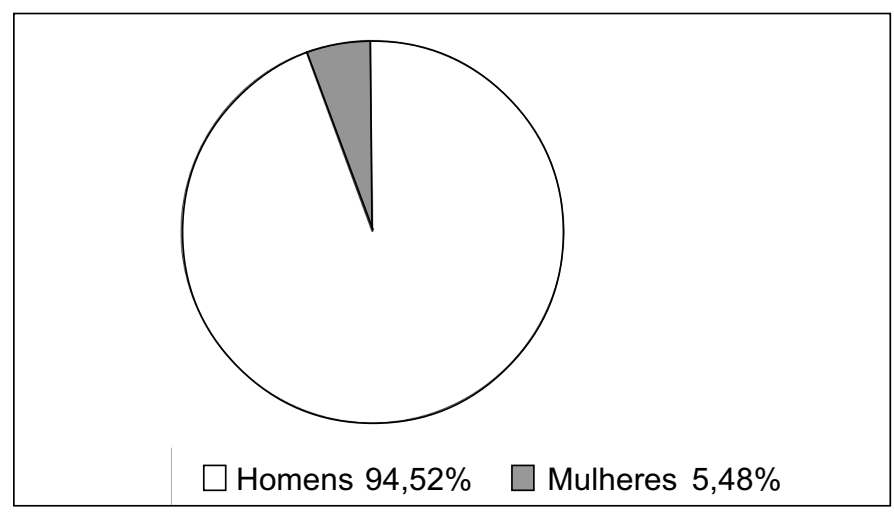

Figura 1 - Distribuição dos pacientes vítimas de queimadura ocular pela cal sem explosão segundo o sexo

\begin{tabular}{|cllc|}
\hline \multicolumn{1}{|c|}{ Tabela 1. Classificação da queimadura química ocular segundo Ballen e Hopper-Hall } \\
Grau & \multicolumn{1}{c}{ Alteração Ocular } & Prognóstico \\
I & Dano epitelial corneano sem isquemia limbar & Bom & Bom \\
II & Córnea levemente opaca sem obscurecer os detalhes da íris e isquemia afetando menos de $1 / 3$ do limbo & Reservado \\
III & $\begin{array}{l}\text { Perda total do epitélio corneano, opacificação corneana causando obscurecimento dos detalhes } \\
\text { da íris e isquemia entre } 1 / 3 \text { e } 1 / 2 \text { do limbo }\end{array}$ & Pobre \\
IV & Córnea opaca obscurecendo a visão da íris e isquemia afetando mais da metade do limbo & \\
\hline
\end{tabular}


O grau de comprometimento ocular deste grupo, pode ser visto na tabela 2 .

Observou-se nos casos de queimadura por "Bomba de Cal" (15-17,05\%) que o sexo masculino foi o mais acometido, sendo $13(86,67 \%)$ homens e $2(13,33 \%)$ mulheres (Figura 2). A faixa etária predominou entre 2 e 20 anos (idade média: 11 anos) (Figura 3), com acometimento bilateral em $8(53,33 \%)$ pacientes e unilateral em 7 (46,67\%); 23 olhos apresentaram lesão ocular. O grau de comprometimento ocular deste grupo, pode ser visto na tabela 3 .

\section{DISCUSSÃO}

Em relação aos pacientes vítimas de queimadura química ocular alcalina (agente cal sem explosão), este trabalho registrou predomínio de indivíduos do sexo masculino $(94,52 \%)$, com faixa etária predominando entre 20 e 40 anos, corroborando com os dados da literatura ${ }^{(1,4,10,20)}$.

A maioria das queimaduras químicas oculares pela cal sem explosão, ocorreu no ambiente de trabalho, dado semelhante aos obtidos na literatura, que relata predomínio de acidentes ocupacionais $^{(1,4,12)}$, e o que evidencia a falta de estrutura e orientação do trabalhador para a prevenção dos acidentes.

Ainda neste grupo, em relação à gravidade e bilateralidade dos casos de queimadura ocular este estudo demonstrou dados similares à literatura, com predomínio de queimaduras grau I $(59,77 \%)$ e unilaterais $(80,82 \%)$ que tem prognóstico bom $^{(7,9,20)}$, confirmando o prognóstico visual da classificação proposta por Ballen e Hopper-Hall.

Já em relação ao grupo da "Bomba de Cal", se a mesma fosse considerada como acidente doméstico, já que é realizada utilizando-se garrafa plástica descartável, água e cal virgem, produtos disponíveis em casa e de fácil acesso a crianças e adolescentes, coincidiria, desta forma, com a literatura estudada

Tabela 2. Distribuição dos olhos afetados pelo agente químico cal sem explosão pela classificação de queimadura química ocular segundo Ballen e Hopper-Hall

\begin{tabular}{ccc|} 
Grau da lesão & Número de olhos afetados & Porcentagem \\
I & 52 & $59,77 \%$ \\
II & 23 & $26,44 \%$ \\
III & 10 & $11,49 \%$ \\
IV & 2 & $2,30 \%$ \\
\hline
\end{tabular}

Tabela 3. Distribuição dos olhos afetados por bomba de cal pela classificação de queimadura química ocular segundo Ballen e Hopper-Hall

\begin{tabular}{ccc} 
Grau da lesão & $\mathbf{N}^{\circ}$ de olhos afetados & Porcentagem \\
I & 5 & $21,74 \%$ \\
II & 6 & $26,09 \%$ \\
III & 3 & $13,04 \%$ \\
IV & 9 & $39,13 \%$ \\
\hline
\end{tabular}

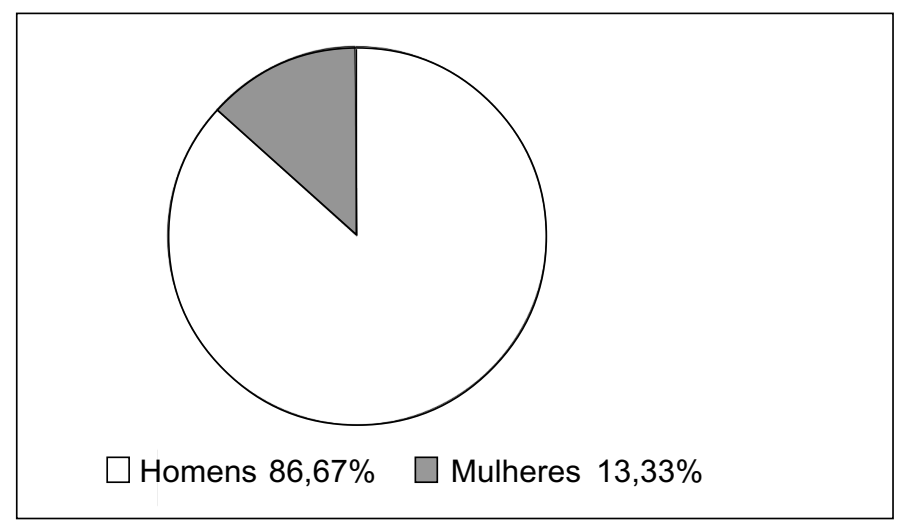

Figura 2 - Distribuição dos pacientes vítimas de queimadura ocular pela "Bomba de cal" segundo o sexo

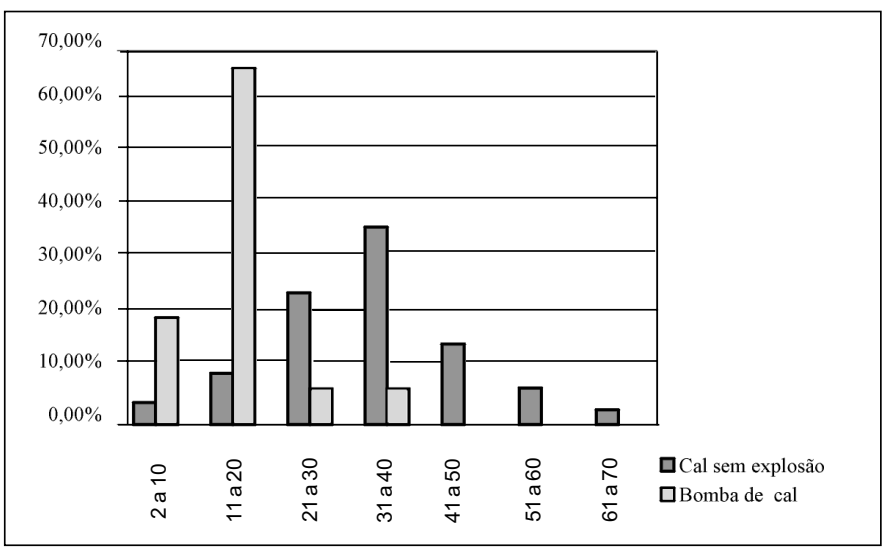

Figura 3 - Distribuição dos pacientes vítimas de queimadura ocular pelo agente químico cal segundo a faixa etária

onde queimaduras químicas oculares em crianças ocorreram na sua maioria em ambientes domésticos acidentalmente ${ }^{(2,7,21-22)}$.

Visto que não há dados na literatura sobre queimadura ocular por "Bomba de Cal", através deste trabalho mostramos nossas estatísticas em relação a esta situação de emergência oftalmológica, onde a "Bomba de Cal" acometeu, na sua maioria, pacientes do sexo masculino, com faixa etária variando entre 2 e 20 anos (idade média: 11 anos), ou seja, as vítimas foram na sua maior parte, crianças e adolescentes. Associado a esse fato, o acometimento ocular destes pacientes foi bilateral em $53,33 \%$ dos casos e com predomínio de queimaduras grau IV $(39,13 \%)$ cujo prognóstico é pobre conforme a classificação proposta por Ballen e Hopper-Hall, o que foi confirmado pela baixa acuidade visual final destas crianças e adolescentes (pior que 20/200) durante o período do estudo.

Em vista do exposto neste estudo, as queimaduras alcalinas oculares devem ser consideradas emergências oftalmológicas devido à sua alta morbidade.

Em relação ao grupo de pacientes vítimas de queimadura ocular pela cal sem explosão, deve haver conscientização por parte das entidades governamentais e trabalhistas quanto à prevenção de acidentes ocupacionais, além da orientação adequada a estes trabalhadores e uso de equipamentos de segurança. 


\section{CONCLUSÃO}

Em vista do exposto neste estudo, as queimaduras alcalinas oculares devem ser consideradas emergências oftalmológicas devido à sua alta morbidade.

Em relação ao grupo de pacientes vítimas de queimadura ocular pela cal sem explosão, deve haver conscientização por parte das entidades governamentais e trabalhistas quanto à prevenção de acidentes ocupacionais, além da orientação adequada a estes trabalhadores quanto ao uso de equipamentos de segurança.

Deve-se dar especial atenção quando as queimaduras químicas oculares são decorrentes da "Bomba de Cal", pois em razão da freqüente bilateralidade, acometimento etário precoce e severidade das queimaduras, levam à severa redução da acuidade visual com indivíduos inativos profissional e pessoalmente antes da máxima produtividade da vida, acarretando em ônus para suas famílias e para a sociedade.

Faz-se necessário o alerta e esclarecimento à população e autoridades, sobre os riscos da "Bomba de cal", pois pelo seu potencial devastador à saúde ocular, a mesma pode desencadear perdas econômicas, sociais e psicológicas às suas vítimas.

\section{ABSTRACT}

Purpose: The assessment of the epidemiological profile of patients victims of lime ocular burns, by the Ophthalmology Department of the Evangelic University Hospital of Curitiba, with emphasis on "Lime Bomb" burn cases. Methods: A study on 88 ocular lime burn patients (lime bomb or not), who came to the HUEC Emergency Service from September 1999 to September 2000. Results: Of 88 patients that were evaluated, $73(82.95 \%)$ had suffered ocular lime burn in their working place and $15(17.24 \%)$ had suffered it due to the "Lime Bomb" itself (local custom). Most of the patients that were examined were males, aged 20-40 years, with predominance of unilateral ocular burn and degree I in case of accident at the workplace, and bilateral and degree IV in case of "Lime Bomb". Conclusion: By comparing the studied groups, it is noticed that those with "Lime Bomb" burn show greater ocular health damage which was demonstrated in this study by means of bilaterality, injury severity and occurring in young patients.
Keywords: Eye burns; Eye/drug effects; Chemical burns; Alkalies/adverse effects; Occupational accidents/prevention \& control; Hospital emergency service; University hospitals

\section{REFERÊNCIAS}

1. Kara José Jr. N, Oliveira Neto JC, Silva ALB, Schellini AS. Acidentes oculares ocupacionais ocorrência em Botucatu, SP, no período de 1988 a 1992. Arq Bras Oftalmol 1994;57:389-93.

2. Moreira ATR, Moreira JR CA. Epidemiologia do trauma ocular na infância. In: Moreira JR. CA, Freitas D, Kikuta HS. Trauma ocular. Rio de Janeiro: Cultura Médica; 1997. cap. 2 (Biblioteca Brasileira de Oftalmologia)

3. Alves MR, Silva MHT, Mattar DB. Trauma químico ocular. In: Alves MR, Kara José N. Conjuntiva cirúrgica. São Paulo: Roca; 1999. cap 19.

4. Andrade AS, Siqueira Bisneto O, Moreira H, Moreira CA. Traumas oculopalpebrais no serviço de pronto-atendimento oftalmológico do Hospital Universitário Evangélico de Curitiba. Arq Bras Oftalmol 1999;62:585-9.

5. Makino MCA, Reggi JRA, Waetge RT, Dantas MCN, Dantas PEC. Perfil das queimaduras oculares na Santa Casa de São Paulo. Arq Bras Oftalmol 1999;62:472.

6. Bison SHDF, Reggi JRA. Traumas oculars: nosologia de 1171 casos. Arq Bras Oftalmol 1995;58:105-11.

7. Noia LC, Araújo AHG, Moraes NSB. Queimaduras oculars químicas: epidemiologia e terapêutica. Arq Bras Oftal 2000;63:369-73.

8. Saari KM, Leinonen J, Aine E. Management of chemical eye injuries with prolonged irrigation. Acta Ophthalmol 1984;(Suppl.161):52-9.

9. Moon ME, Robertson IF. Retrospective study of alkali burns of the eye. Aust J Ophthalmol 1983;11:281-6.

10. Klein R, Lobes Jr LA. Ocular alkali burns in a large urban area. Ann Ophthalmol 1976;8:1185-9.

11. Souza RL, Nassarala JJ. Incidência de queimadura ocular em um centro de referência do centro-oeste. [resumo] Arq Bras Oftal 1997;60:376.

12. Morgan SJ. Chemical burns of the eye: causes and management. Br J Ophthalmol 1987;71:854-7.

13. Freitas D. Queimadura ocular. In: Moreira Jr. CA, Freitas D, Kikuta HS. Trauma ocular. Rio de Janeiro: Cultura Médica; 1997. cap 1. (Biblioteca Brasileira de Oftalmologia)

14. Arffa RC. Traumatismos corneales: queimaduras químicas. In: Gryson M. Enfermedades de la cornea. $2^{\text {th }}$ ed: México: Médica Panamericana, 1985. p.579-85.

15. Mc Culley JP. Chemical injuries. In: Smolin G, Thoft RA, editors. The Cornea: Scientific Foundation an Clinical Practice. 2nd. ed. Boston: Little, Brown; 1987. p.527-42.

16. Wagoner MD. Chemical injuries of the eye: current concepts in pathophysiology and therapy. Surv Ophthalmol 1997;41:275-313.

17. Paterson CA, Pfister RR, Levinson RA. Aqueous humor PH changes after experimental alkali burns. Am J Ophthal 1975;79:414-9.

18. Brown SI, Weller CA, Wassermann HE. Collagenolitic activity to alkali burned corneas. Arch Ophthal 1969;81:370-3.

19. Gerard M, Merle H, Ayeboua L, Richer R. Prospective study of eye burns at the Fortde France University Hospital. J Fr Ophthalmol 1999;22:834-47.

20. Davis AR, Ali QH, Aclimandos WA, Hunter PA. Topical steroid use in the treatment of ocular alkali burns. Br J Ophthalmol 1997;81:732-4.

21. Kersjes MP, Reifler DM, Murer JR, Trestail JH, McCoy DJ. A review of chemical eye burns referred to the Blodgett Regional Poison Center. Vet Hum Toxicol 1987;29:453-5

22. Kara-José N, Alves MR. O trauma ocular como causa de cegueira no Brasil. In: Moreira Jr. CA, Freitas D, Kikuta HS. Trauma ocular. Rio de Janeiro: Ed. Cultura Médica; 1997. cap. 1. (Biblioteca Brasileira de Oftalmologia).

\title{
ABO ELETRÔNICO
}

\author{
Novo site
}

\section{Acesso: http://www.abonet.com.br}

